\title{
Diseases Diagnosis Using Medical Palmistry Fuzzy Model
}

\author{
Zainab Othman ${ }^{1, a}$ and Sarmad Saleem ${ }^{1}$ \\ 1Department of Computer Science, Science College, University of Basra, Iraq.
}

\begin{abstract}
The design n, implementation, and use of biomedical information systems in the form of computer - aided decision support have become essential and widely used over the last two decades. Medical decision support systems play an increasingly important role in medical practice by assisting physicians who make clinical decisions. Medical scientists discovered that the hand can be used as an indicator for medical problems and the palm is the reflection of activities going on brain. The purpose of this research is to design and implement a decision support model for healthcare on the basis of medical palmistry to diagnose the diseases from palm colors. Database palm images for patients infected with specific disease is created from capturing live images from hospitals. Digital image processing techniques on input images are applied. Fuzzy Inference System is used to present medical knowledge as network diseases connected with each other by logical relations. The model is built to assist medical practitioners for taking diagnosis decision for four special diseases. The results obtained from this work are confidence.
\end{abstract}

\section{Introduction}

Computer - aided diagnosis is rapidly became a part of the routine clinical works for the detection of disease. The computer output is used as a "second opinion" in assisting for diagnosis a different diseases. Generally the proposed algorithm consists of many procedures that may include image feature analysis, and data classification via the use of tools such as fuzzy logic or artificial neural network (ANN) which are referred to as "artificial intelligence" [1]. One of the fields to identify disease is analysis of the color of human palm. Medical science studies the palm for various coloration of different region to get assistance in decision making. Different colors existing at different regions in palm are based on blood circulation at that region as well as presence of disease in human body. The computer will give more accurate results than human vision, because it overcomes the limitations of human eye like subjectivity and resolution power [2]. There are many previous attempts had been done to diagnose the disease from palm. But the researches in this field are still in the beginning.

B. Shanmugapriya et al [3]. Presented about the handling of valuable information from human hand image in various fields like biometrics, prophesy and medical diagnosis. A.Navpat et al [4]. Discussed the design of a system that helps in predicting the palmistry details by scanning an image of the palm. H. Pandit et al [5]. Explained a prototype for human

Palm based on digital image processing and analysis in field of healthcare. D. Desai et al [6].Applies digital image processing techniques on input palm images to identify certain features in the image and by using knowledge base of medical palmistry it analyzes certain features in image and predicts probable disease. In this paper new Fuzzy model is proposed to identify certain features in the palm images depending on knowledge base of medical palmistry. Database for live palm images had been created from Basra hospitals. This database contained palm images for patients had diseases in heart, kidney, lung, and obstruction in the circulation of the blood.

\section{Palmistry Basics}

Medical science has found that the palm contains more nerves cells than any other portion of the nervous system. So that, palm is the reflection of activities going on in brain. Thus, whatever is indicated in palm is result of biological and psychological changes in the human system [7]. A normal palm has color a light red color or a pinkish red with a shiny and smooth texture. While the following colors denote there are abnormal conditions [8].

\footnotetext{
a Corresponding author: zai_nab_alfahad@yahoo.com
} 
a. Pale White: A palm appearing pale white in color indicates anemia or possibly occult bleeding.

b. If the palm looks white, this usually indicates lung disease or inflammation in the body.

c.Blue: A Blue palm usually indicates intestinal obstruction.

d. Dark Green: A Palm with a dark green color usually indicates obstruction in the circulation of the blood.

e. Yellow: A sallow yellow palm usually indicates chronic disease. This is because chronic disease typically affects the spleen and stomach. A palm that looks yellowish brown and has no sheen indicates the possibility of cancer.

e. Black Red: If the whole palm is covered with dark red or purple spots, this is usually seen in liver disease

f. Purple: When the subcutaneous tissue of the palm shows prune colored purple, it indicates serious infectious shock.

g. Grey: Thin Cigarette-ash like spots on the palm

h. Black Brownish: A palm that looks Brownish

is often seen in kidney disease.

\section{Database for Color Palm Images}

Palmistry technique acts as one of the aid tools for diagnosing purposes. The Internet does not have like database for color palm images influenced with disease. Then to collect diseased palm images, it is practically to get them live from patients in hospitals. This caused a big problem, because most patients don't cooperate in this project. Also official approvals were difficult and take long time.

The specific Basra hospitals departments had been visited which are related with this work and a collection of color palm images are accumulated. The database includes images of normal persons and patients between the ages of 20 and 80 years. About $20 \%$ percentage of images are taken from female and the rest are for male. The images are captured for right and left palms. This operation built a Database for normal and diseased color palm images.

\section{Limitation in Capturing Palm Images}

Mainly there are two capturing devices. First, CCD based scanner and the second, is digital camera. The CCD based scanner device is not available in Basra hospitals, so the digital camera is the only solution to capture palm images. In this work, samples of palm images are digitally captured under standard and control environment. So many limitations are considered to capture a palm image using Digital Camera. These limitations are:

a. Image capturing is at the morning.

b. The light intensity must be controlled to suitable value.

c. The camera was placed at a distance of 40 to 50 $\mathrm{cm}$ directly above the hand palm of respondent.

Special Black Board is made to locate the palm on it before capturing the image. Figure (1) shows the black board using with capturing palm images. Some patients refused to put their hands on this black board. So black cushion was used for this purpose.

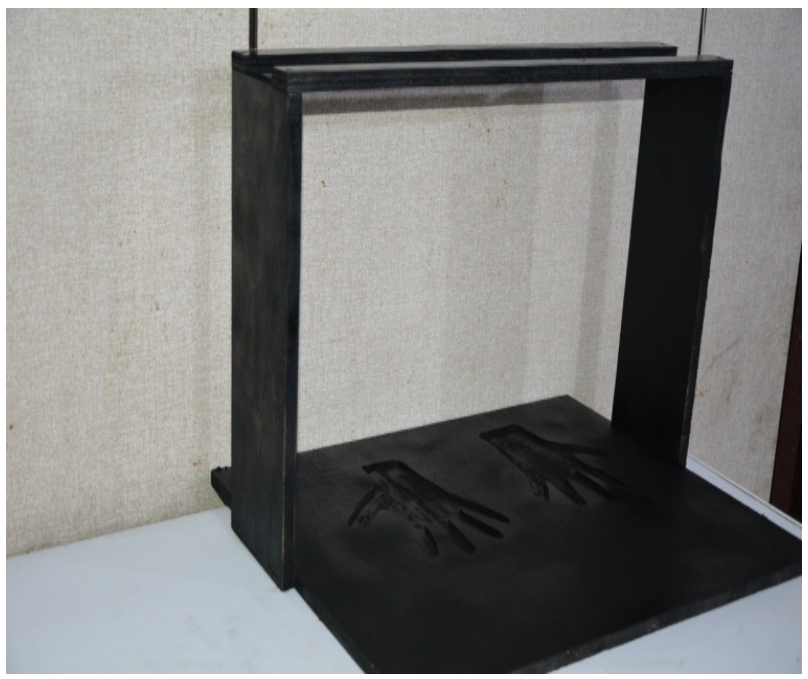

Figure 1. Black Board used in Capturing Color Palm Image.

\section{The Proposed Model}

The proposed model structure is designed to have the following architected as shown in Figure (2).

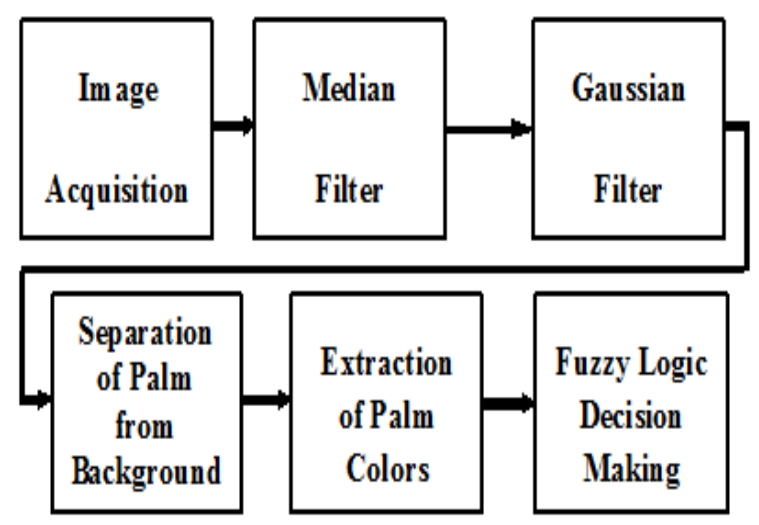

Figure 2. Block Diagram for Proposed Model

\section{Image Acquisition}

Digital camera of type Nikon d7100 with 24.1 megapixel is used to collect palm images from patients. 
More than 500 images are captured from patients and only 436 obviously of them are used in this paper. Four diseases are concerned in this paper:

a. Lung disease.

b. Obstruction in the circulation of

The blood.

c. Kidney disease.

d. Heart disease.

The RGB image for size $12 \mathrm{M}$ Byte is formatted and saved as jpg format. The total capacity of the obvious palm images is more than $5 \mathrm{G}$ byte. Figure (3) shows sample of color palm images.

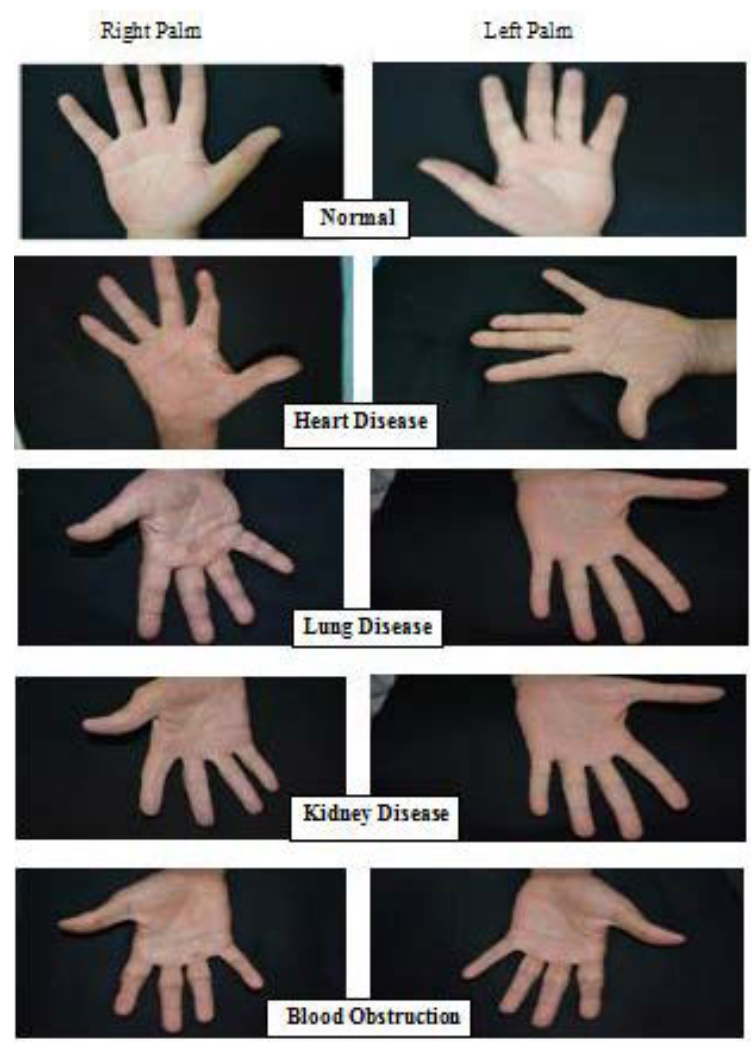

Figure 3. Samples of Palm Images

\section{Filtering Results of Median}

Median filter is a denoising filter used to remove salt and pepper noises from digital image [9]. The Median Filter is a two dimension filter, so it can't use for color image. To avoid this problem the color image is extracted to the individual red, green, and blue channels. In the RGB color model, each color appears in its primary spectral components of red, green, and blue. Each color is treated alone by Median Filter and then reconstruction is used to produce denoised free image.

Different window sizes are taken, but the best result is obtained when the window size is $3 \times 3$. The algorithm of Median Filter is shown as below:
Algorithm : processing median filter

Input: RGB Color Palm Image

Output: Denoising RGB Color Palm Image

Begin

1. read $R G B$ Palm Image image=imread(C:|palm1.jpg)

2. extract the individual red,green and blue color channels

redchannel $=$ image $(:,:, 1)$

greenchannel=image(:;:,2)

bluechannel=image(:,:,3)

3. median filter the channels

redmf=medfilt2(redchannel,[3 3])

greenmf $=$ medfilt2(greenchannel,[3 3])

bluemf=medfilt2(bluechannel,[3 3])

4. reconstruct the denoise free $R G B$ image

image $=$ cat $(3$, redmf,greenmf,bluemf $)$

End.

Median Filtering have a good edge preserving ability, and does not introduce new pixel values to the processed image. As well as Median Filter reduces the blurring of edges. Median Filter reduces the blurring of edges. Figure (4) presents the operation of the Median Filter for a sample of palm images.

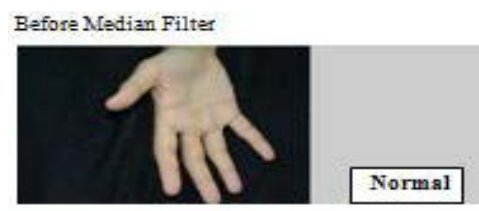

After Median Filter
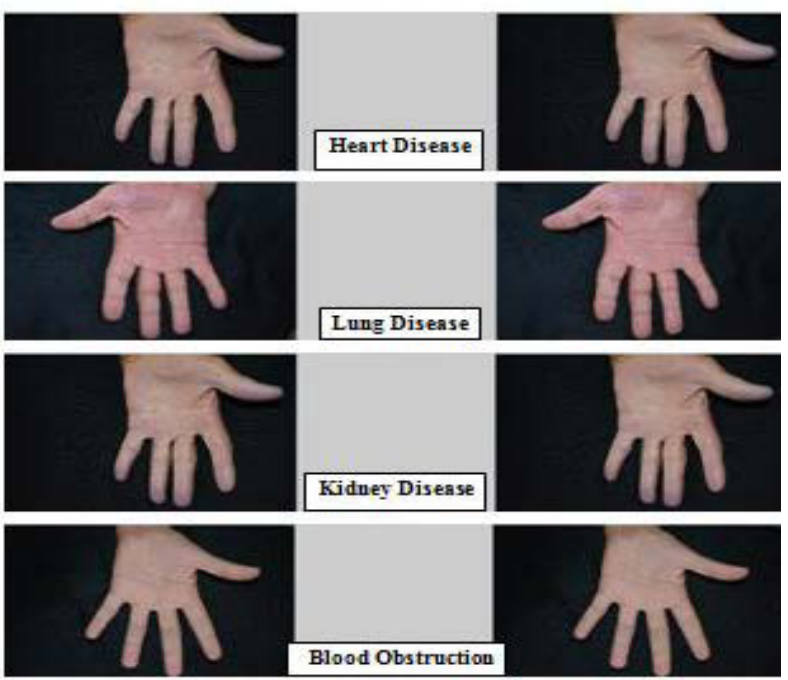

Figure 4. Output Images from Median filt 


\section{Filtering Results of Gaussian}

One kind of noise which occurs in all recorded images to a certain extent is detector noise and visible as grains in the image. It may arise in the image as effects of basic physics-like photon nature of light or thermal energy of heat inside the image sensors [10]. The output images from Median Filter are used as input to Gaussian Filter. Figure 5 shows samples of images processing by Gaussian Filter. The Algorithm used to process Gaussian Filter is shown as below:

\section{Algorithm : processing Gaussian filter}

Input : RGB Color Palm Image after Processing by Median filter

Output : Denoising RGB color Palm Image

Begin

1. Get RGB Palm Image from Median filter Image $1=$ image

2. Processing image by Gaussian filter $\boldsymbol{h}=$ fspecial('gaussian',[7,7],3) image $=$ imfilter $($ image $1, h)$

End.

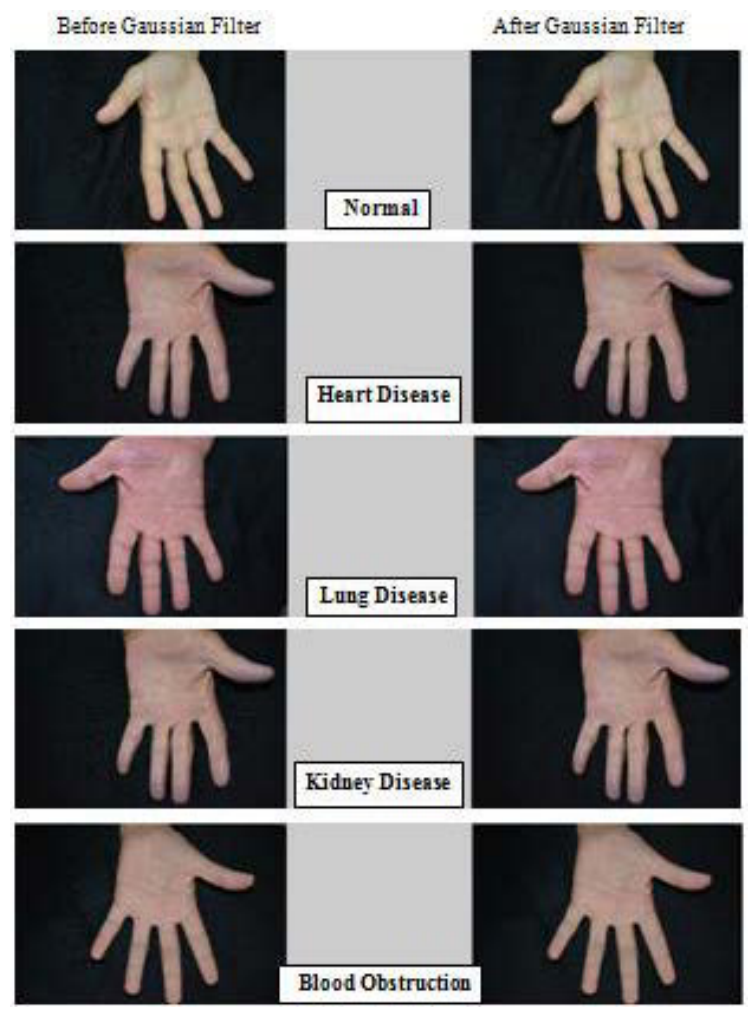

Figure 5. Output Images from Gaussian filter

\section{Separation Palm from Background}

The main difficulty with trying to separate an image from its background is that there are pixels which cover an area of the picture which is partly Foreground and partly background. With any of the methods, a pixel must be declared whether either foreground or background, even though it may be both. Threshold is actually a pretty good method to extract palm from background.

Actually all palm images are captured using black fixed background. But this operation is no sufficient to separate the palm image from background. So threshold method is used for this purpose. In the palm image of human, by eye's observation of RGB of palm. It is easy conclude that, red component having higher value than green and blue components. This observation is used as criteria to store all those pixels that satisfy the above condition. Figure 6 shows samples of images after separation processing. The following is Matlab code used in separation process.

\section{Algorithm : separation Palm from Image}

Input : Get denoising Image from Gaussian filter

Output : Palm Image separation from background

Begin

Image $1=$ image

1. Get the image dimension number of color bands should be $=3$

[rows, columns, number of Color bands J=size(image)

2. Extract the individual red, green, blue color Channels

Redchannel $=$ image $(: ;, ; 1)$

Greenchannel $=$ image $(: ;, ; 2)$

Bluechannel $=$ i mage $(: ;: ; 3)$

3. Separate background from palm image using threshold

$k=0$

For $I=1$ :rows

For $j=1:$ columns

If redchannel $(i, j)>40 \mid$ |greenchannel $(i, j)>40$

||bluechannel $(i, j)>40$

$k=k+1$

Else

Redchannel $(i, j)=0$;greenchannel $(i, j)=0$; bluechannel $(i, j)=0$

end

end

end

4. Reconstruct the free RGB image after separation from background

Image $=$ cat $(3$, redchannel,greenchannel, bluechannel)

End. 


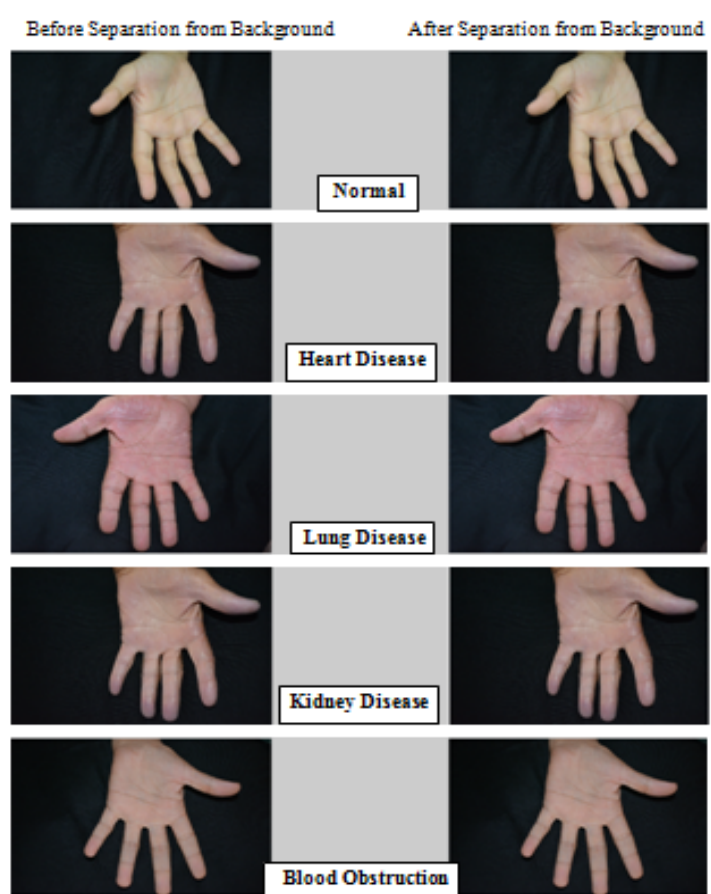

Figure 6. Images after Separation processing

\section{Extraction of Palm Colors}

Color images have multiple color intensity values stored per pixel. Different colors are generated by combination of red, green, and blue colors. In this model four diseases are chosen for detection. These diseases are chosen depending on available database .The chosen diseases are:

1. Lung disease: this disease causes the palm to get Pale White color with RGB value around [248,248,255]. I.e. High red, high green, and high blue.

2. Obstruction in the circulation of the Blood: this disease paints palm with dark green color and RGB value almost $[0,100,0]$. I.e. Low red, medium green, and Low blue.

3. Heart Disease: palm has grey color when the human is infected with this disease and the RGB value is about $[128,128,128]$. i.e. medium red, medium green, and medium blue.

4. Kidney Disease: this disease infects the human and produces a nearly brownish color with RGB value less than $[160,42,42]$. i.e. medium red, low green, and low blue.

\section{Design of Fuzzy Inference System}

There are two types of Fuzzy Inference Systems (FIS):
(a) Mamdani.
(b) Sugeno.

The color of the normal palm is light red or pinkish and RGB value is concerning [255, 80,150], i.e. high red, low green, and medium blue. Note that, these values of RGB are taken from RGB color codes chart presented in Internet web sites. These two types of inference systems vary somewhat in the way outputs are determined .Mamdani-type inference, as defined for the toolbox, expects the output membership functions to be fuzzy sets. After the aggregation process, there is a fuzzy set for each output variable that needs defuzzification. While Sugeno-type systems can be used to model any inference system in which the output membership functions are either linear or constant [11].

In this paper Mamdani-type inference system is used to diagnostic system with three inputs and single output. Also four rules are suggested each rule is noted to specific disease. Figure (7) shows the design of FIS in Matlab.

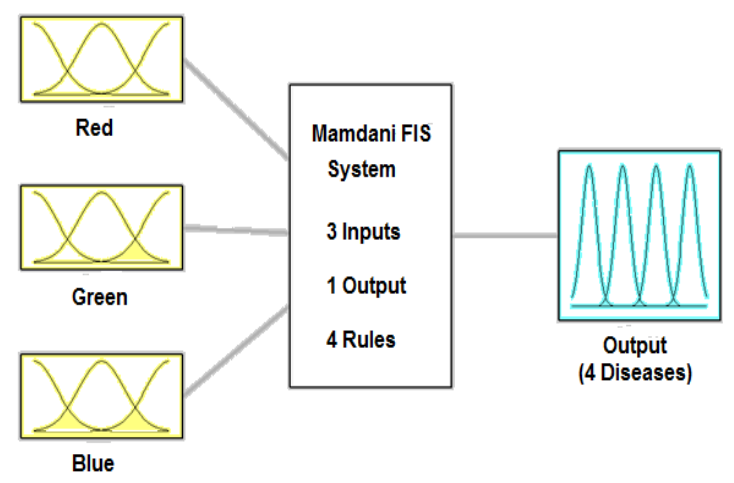

Figure 7. Matlab FIS for Diagnosis System.

Membership functions are used in the fuzzification and defuzzification steps of a FIS, to map the non-fuzzy input values and output value to fuzzy linguistic terms. An important characteristic of fuzzy logic is that a numerical value does not have to be fuzzified using only one membership function. In other words, a value can belong to multiple sets at the same time.

So color values can be considered as "High", "Medium", and "Low" at the same time with different degree of memberships. The most common types of membership functions are triangular, trapezoidal, and Gaussian shapes. These three shapes $(a, b, c)$ are shown in Figure (8) and considered in this paper. 


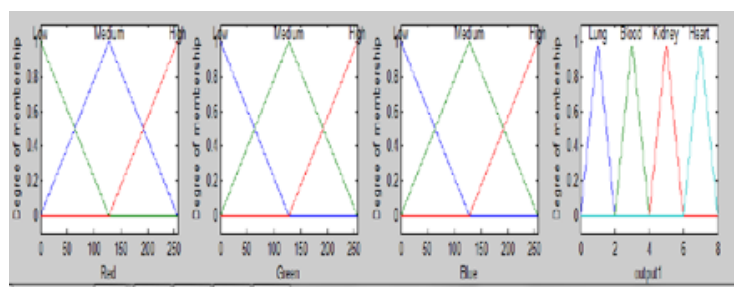

(a) Triangular Membership.

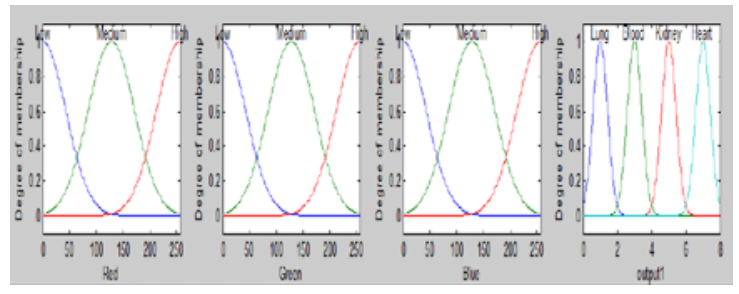

(b) Gaussian Membership.

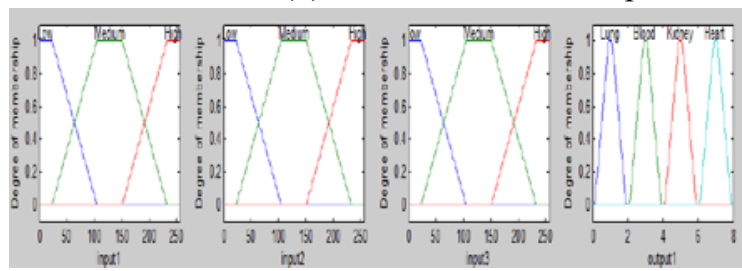

(c) Trapezoidal Membership.

Figure 8. Memberships Used in the Proposed Model.

In a FIS, a rule base is constructed to control the output variable. A fuzzy rule is a simple IF-THEN rule with a condition and a conclusion. In Table 1, fuzzy rules for FIS is presented.

Table 1. Fuzzy Rules for Diagnostic System.

\begin{tabular}{||l||}
\hline Fuzzy Rules \\
2- IF (Red is Low) and (Green is Medium) and \\
(Blue is High) then (Output is Lung) \\
3- IF (Red is Medium) and (Green is Low) and \\
(Blue is Low) then (Output is Kidney) \\
4- IF (Red is Medium) and (Green is Medium) \\
and (Blue is Medium) then (Output is Heart)
\end{tabular}

Table (2) shows the matrix representation of the fuzzy rules for the used FLS. This matrix is $3 \mathrm{D}$ matrix, but it is converted into $2 \mathrm{D}$ for simplicity.
Table 2. Fuzzy Matrix for Diagnostic System.

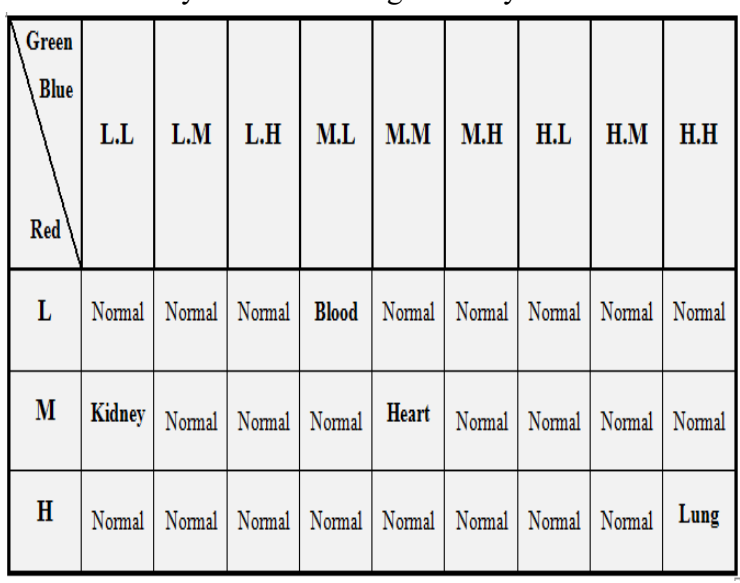

The inference procedure is called as the "compositional rule of inference". The inference is determined by two factors; "implication operator" and "composition/aggregation operator". In Mamdani : Mamdani implication is min for each rule:

Rule1 $\mathrm{ml}=\min \{\mu \operatorname{red}(\mathrm{x}), \mu \operatorname{green}(\mathrm{x}), \mu$ blue $(\mathrm{x})\}$

Rule2 $\mathrm{m} 2=\min \{\mu \operatorname{red}(\mathrm{x}), \mu \operatorname{green}(\mathrm{x}), \mu$ blue $(\mathrm{x})\}$

Rule $3 \mathrm{~m} 3=\min \{\mu \operatorname{red}(\mathrm{x}), \mu \operatorname{green}(\mathrm{x}), \mu$ blue $(\mathrm{x})\}$

Rule4 $\mathrm{m} 4=\min \{\mu \operatorname{red}(\mathrm{x}), \mu \operatorname{green}(\mathrm{x}), \mu$ blue $(\mathrm{x})\}$

Mamdani aggregation is $\max$ for aggregation four outputs:

$\operatorname{output}(\mathrm{z})=\operatorname{Max}\{\mathrm{m} 1, \mathrm{~m} 2, \mathrm{~m} 3, \mathrm{~m} 4)$

Figure (9) shows the Rule viewer in FIS:

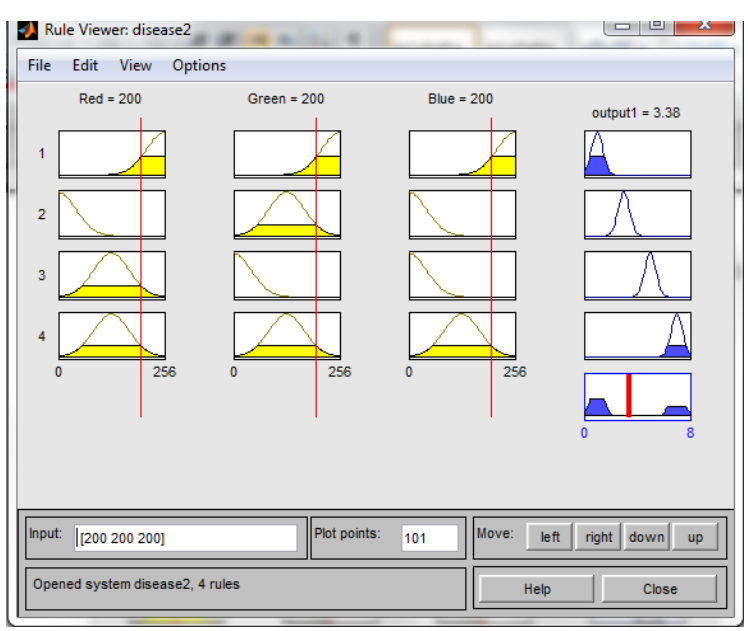

Figure 9. Shows the Rule viewer in FIS:

There are different algorithms for defuzzification. The purpose is to obtain a crisp value, represented with a dot in the figure, from this fuzzy result. 
One of the most common is the center of gravity (or center of area).

$$
C O G(N)=\frac{\sum_{i=1}^{k} x i \mu(x i)}{\sum_{i=1}^{k} \mu(x i)}
$$

Where

xi specifies element of output universal set, $\mu(x i)$ represents the membership degree of element xi, and $\mathrm{k}$ specifies the number of elements in the universal set.

From FIS the results of disease diagnosed are obtained. The Gaussian Membership gives the best results. Table (3) presents the results obtaining from this membership.

Table (3). FIS Results using Gaussian Membership.

\begin{tabular}{|c|c|c|c|c|c|c|c|}
\hline \multicolumn{2}{|c|}{ Actual values } & \multicolumn{5}{|c|}{ Predicted Values from Model } & \multirow[b]{2}{*}{ Accuracy } \\
\hline Disease Type & $\begin{array}{l}\text { No. of } \\
\text { Live } \\
\text { Images }\end{array}$ & $\begin{array}{c}\text { As } \\
\text { Heart }\end{array}$ & $\begin{array}{l}\text { As } \\
\text { Lung }\end{array}$ & $\begin{array}{c}\text { As } \\
\text { Blood } \\
\text { Obstruction }\end{array}$ & $\begin{array}{c}\text { As } \\
\text { Kidney }\end{array}$ & As Normal & \\
\hline Heart & 140 & 135 & 0 & 0 & 0 & 5 & $96 \%$ \\
\hline Lung & 48 & 24 & 18 & 0 & 0 & 6 & $37.5 \%$ \\
\hline Blood Obs. & 56 & 20 & 0 & 23 & 0 & 3 & $50 \%$ \\
\hline Kidney & 92 & 43 & 8 & 2 & 31 & 8 & $33.6 \%$ \\
\hline Normal & 100 & 17 & 3 & 0 & 0 & 80 & $80 \%$ \\
\hline
\end{tabular}

\section{Performance of Proposed System}

In order to investigate the performance of the proposed model, the actual number of each disease is presented with these diagnosed by the proposed model. The comparisons between actual results and
Predicted results are presented in Figure (10), Figure (11), and Figure (12) using Triangular, Gaussian, and Trapezoidal Memberships respectively. The figures show the accuracy of different memberships.

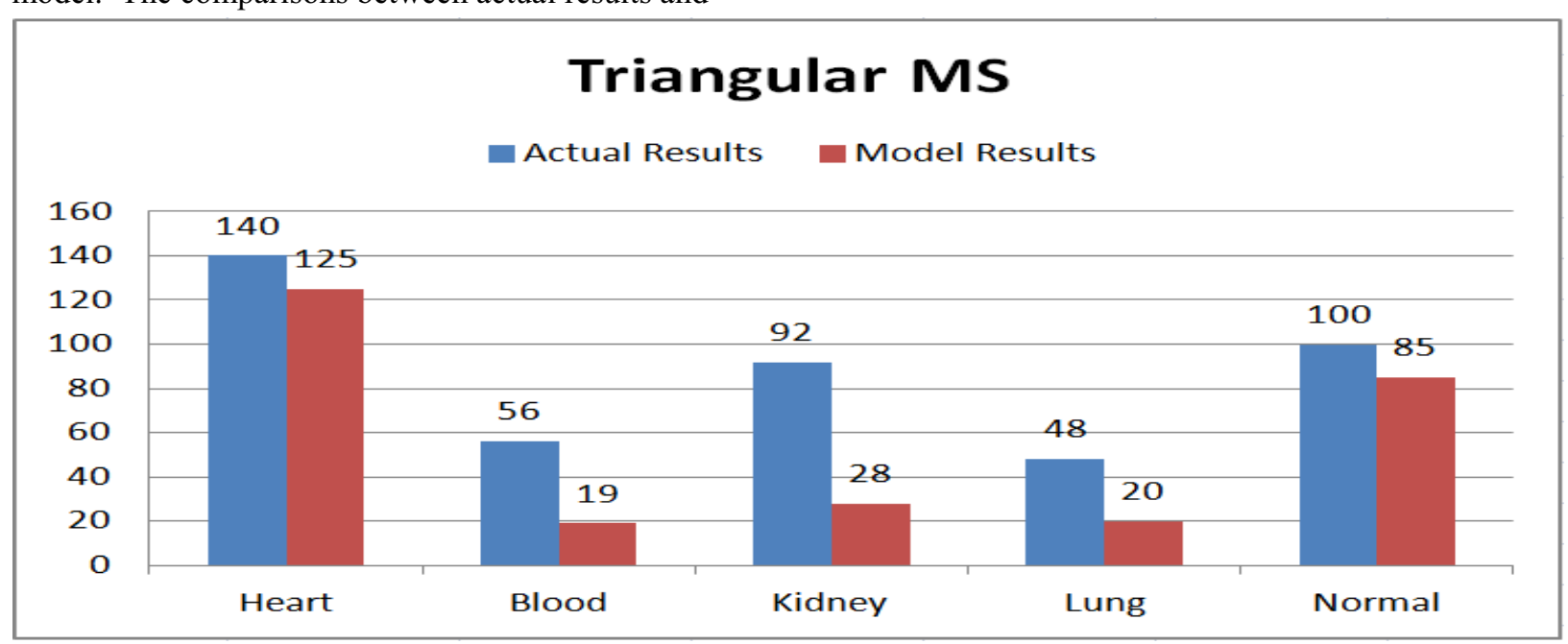

Figure 10. Results obtained using Triangular Membership 


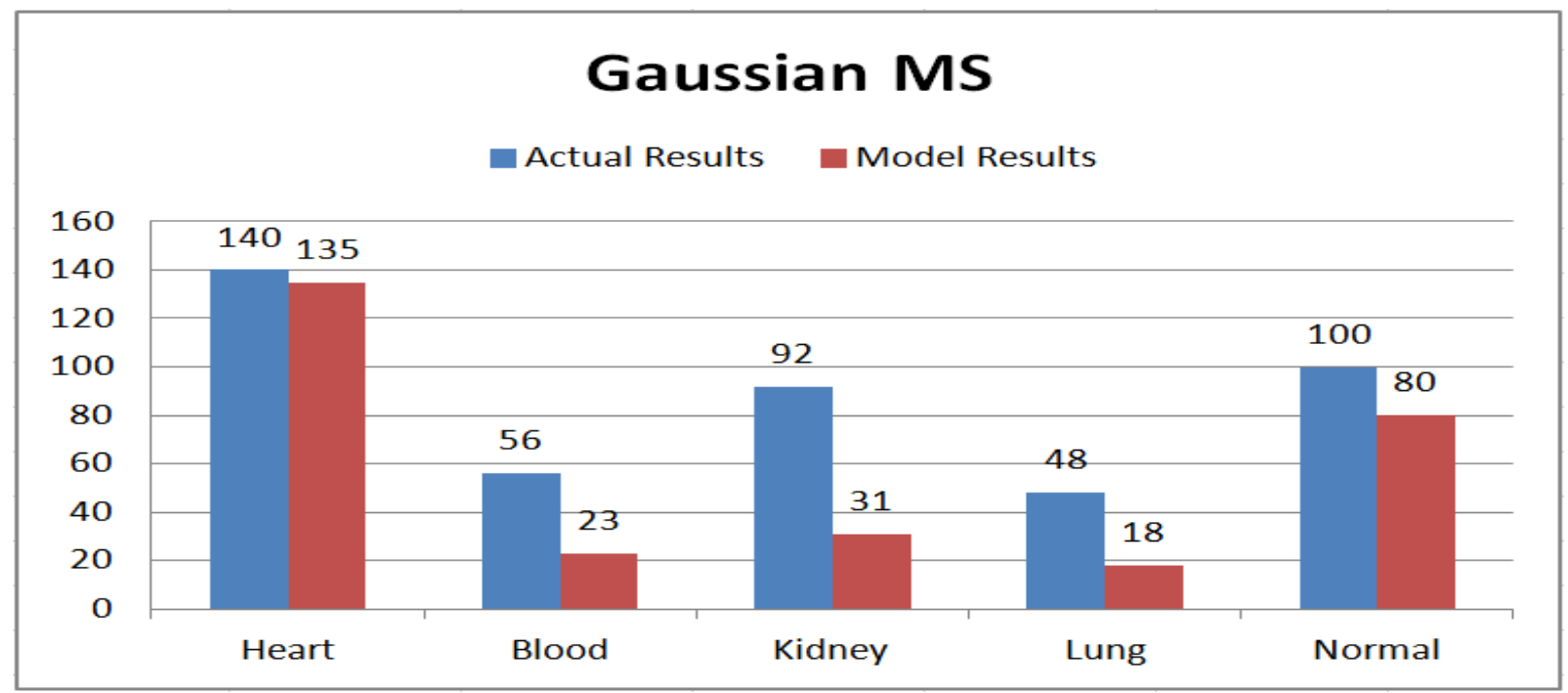

Figure 11. Results obtained using Gaussian Membership.

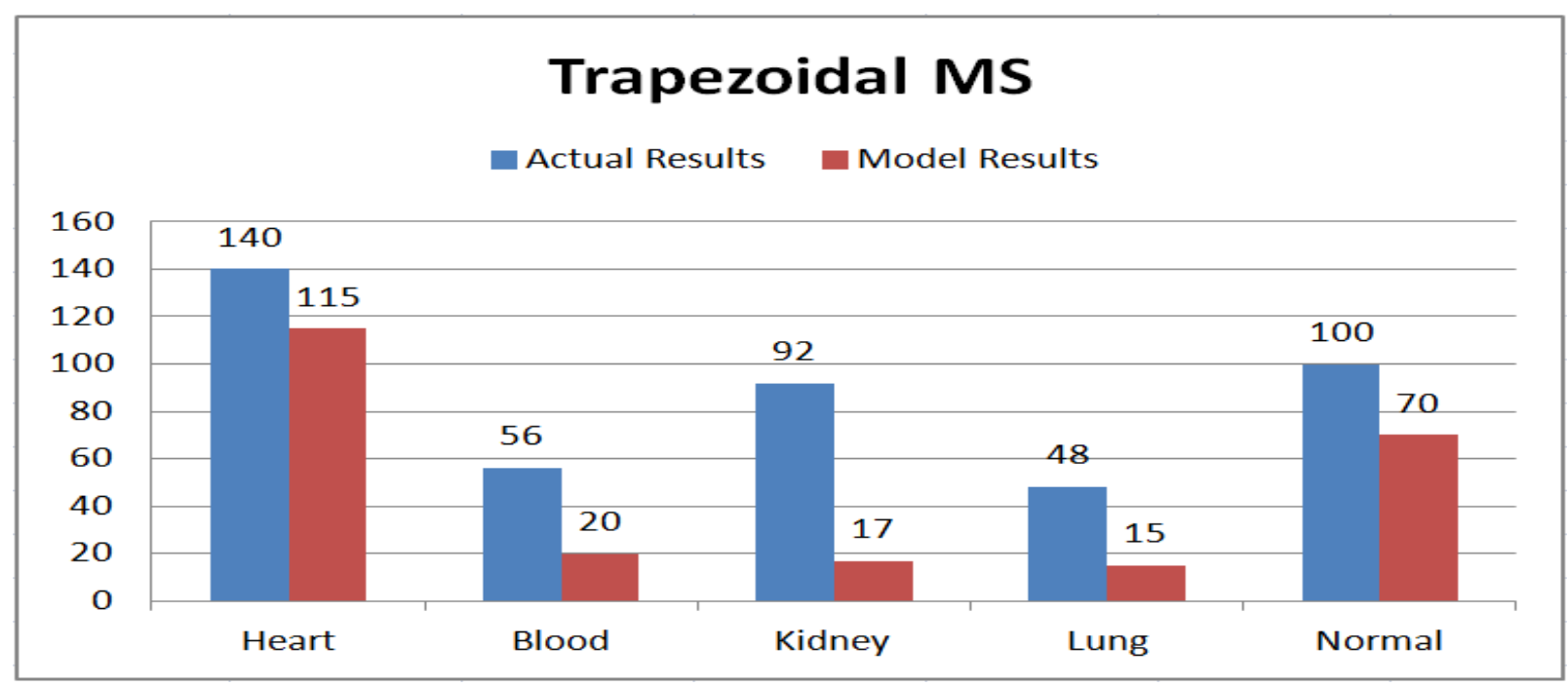

Figure 12. Results obtained using Trapezoidal Membership.

From these results, the following points are important to focus on:

1. Using Gaussian Membership in input and output of FIS gives more accurate results from Triangular and Trapezoidal Memberships.

2. Diagnosis of Heart disease in this Model have highest accuracy than the other diseases.

3. When Some Normal cases are diagnosed in this Model, it gives Heart disease. This happen because Normal cases are collected for persons they believe are Normal persons but perhaps there are such disease without they know that.

4. It is impossible to diagnosis more than one disease from single color palm image.

5. Most diseased palm images (about $80 \%$ of them) are captured for old ages patients. So most of them are infected with Heart disease and another disease. The proposed Model can specify only one disease from single palm image, so the Heart disease appears on the palm rather than other disease. i. e. Heart disease influence palm color instead on the any other disease.

6. The accuracy results for diagnosis of Lung, Kidney, and Obstruction in the Circulation of Blood diseases can be increase if the collection of their images from young patients have not Heart disease but are infected with one these diseases.

7. There are not study in this field obtaining final results, so comparison between this work and the other study is not existed.

8. The performance of proposed Model is confidence and the Model will give more accurate results if database of collection color palm images is increased with more and more images. 


\section{Conclusions and future works}

The medical diagnosis model is built to assist medical practitioners for taking diagnosis decision. So, the final and important decision is related to doctor. Many conclusions are obtained from this work the chosen of excellent image acquiring device is necessary in capturing obvious palm image. The CCD scanner device is very important in capturing color palm image. So, Basra hospitals must advice them to buy this device. Also, denoising filters are important to get better extraction features from image. The filter must be chosen very careful in order to prevent the destroying the palm information.Using expert system like Fuzzy Logic System is significant in decision. These expert systems have ability to give less risk decision. In the end medical palmistry field needs more discuss and investigation.

In the future there are many interesting works that can be suggested in this field. As example, we mention the Palm image can be segmented corresponding to its mounts and then enlarged to get better observation .therefore, Implement a computer program to automatically search the palm image to indicate the symbols in the palm.we can Build large database for palm image infected with diseases and investigate the other diseases related to palm color and palm symbol. Finally The system can be developed by focusing on the palm lines which contain rich information and can be used to get more accurate diagnosing decisions.

\section{References}

1. J. Shiraishi, Q. Li, D. Appelbaum, and K. Doi , "Computer-Aided Diagnosis and Artificial Intelligence in Clinical Imaging", Seminars in Nuclear Medicine, Elsevier Inc, Vol. 41, No. 6 (2011)

2. H. Pandit and D. Shah, " The Model of nail color analysis - An application of Digital Image Processing", International Journal of Advanced Research in Computer Science and Software Engineering, ISSN: 2277-128, Vol. 3, Issue 5, May (2013)

3. B. Shanmugapriya and R. Rajesh, "Survey: Applications of Bravura Information in Human Hand", International Journal of Wisdom Based Computer, Vol. 1(3) (2011)

4. A. Navpat, R. Mukherjee, V. Pandita and S. Gupta, "Application of Prediction Software in Palmistry", MPGI National Multi Conference, Proceeding Published by International Journal of Computer Applications ISSN: 0975-8887 (2012)

5. H. Pandit and D. Shah, "A System for Palm Color Analysis Healthcare", International Journal of
Advanced Engineering Technology, E-ISSN: 09763945, Vol. V, Issue I (2014)

6. D. Desai, M. Parekh, D. Shah, V. Sawant, and A. Nagare, "Automated Madical Palmistry System Based on Image Processing Techniques", International Journal of Advanced Research in Computer Science and Software Engineering, Vol. 5, Issue 1, ISSN: 2277-128X (2015)

7. F. Ahamad, K. Jhansi and S. Varali, "Traditional Chinese Medicine and Its Significance", Medicinal Chemistry, ISSN: 2161-0444, Vol. 5, Issue 2 ( 2015)

8. X. Zong and G. Liscum - "Chinese Medical Palmistry-Your Health in Your Hand", Blue Poppy Press, ISBN: 0-938185-64-3 (1995)

9. R. Khanna, "A Review of Image Restoration Techniques for Salt and Pepper Noise with Test Results", IJARECE, ISSN: 2278 - 909X, Vol. 4 Issue 2 (2015)

10. M. Huber, Nonlinear Gaussian Filter: Theory Algorithms and Application, Habilitation, Karlsruher Institut für Technologie, Publish by (KIT) Fakultt für Informatik, (2015)

11. N. Mishra, "A Reviw on the Applications of Fuzzy Expert System for Disease Diagnosis", International Journal of Advanced Research in Engineering and Applied Sciences, ISSN: 22786252, Vol. 3, No. 12 (2014) 